\title{
Mathematics of uncertainty: an exploration on semi-elliptic fuzzy variable and its properties
}

\author{
Palash Dutta ${ }^{1}$ (B)
}

Received: 16 September 2019 / Accepted: 9 December 2019 / Published online: 19 December 2019

(c) Springer Nature Switzerland AG 2019

\begin{abstract}
Uncertainty is more or less encountered in industrial and medical systems as well. Uncertainty theory is an upgraded theory which comprises of possibility measure, necessity measure and credibility measure plays significant role in modelling uncertainty. In connection with uncertainty modeling, a special and intricate fuzzy variable viz., semi-elliptic fuzzy variable (SEFV) is thrashed out here. Subsequently, an attempt has been made to derive possibility, necessity and credibility measure of the SEFV first. Later, some other properties such as expected value, variance, rational upper bound etc are presented and based on that mean and variance ranking of SEFVs are proposed. Afterwards reliability analysis and medical diagnosis cases are carried out which exhibit the efficiency and novelty of the derived SEFV. In this work done, it is observed that the present work has the capability to resolve problems under uncertain complex situations.
\end{abstract}

Keywords Uncertainty · Fuzzy variable · Semi-elliptic fuzzy variable Possibility measure · Necessity measure . Credibility distribution

\section{Introduction}

Uncertainty occurs due to lack of precision, deficiency in data, diminutive sample sizes, foreseeable man-made/artificial mistakes etc., is an unavoidable component of real world problems. To deal with this type of uncertainty fuzzy set theory (FST) [1] is explored. In power system planning reliability investigation is an extremely significant feature. The electrical energy production and consumption are the essential operating characteristic of the power system those are operated simultaneously and consequently, the investigation of reliability obligation for power system is incredibly elevated. Generally probabilistic approaches of reliability investigation are explored. However, due to association of uncertainty in the system classical probability approaches are seemed to be inappropriate and subsequently, fuzzy reliability investigation models are taken into consideration [2]. Some recent applications in reliability investigations can be found in [3-15]. On the other hand, in medical diagnosis, usually a disease is characterized by several unswervingly perceptible symptoms which persuade the patient to visit a consultant or practitioner. A set of clinical inspections are commenced to make out the incidence of a disease. In the sphere of medical diagnosis, plenty of variables persuade the decision making process and subsequently, discriminate the judgments of the consultant or practitioner. Furthermore, mainly the medical diagnosis quandary engages dealing with uncertainties and so needed to integrate all the information into investigation. Therefore, fuzzy sets are explored to represent uncertainty and to perform medical diagnosis as well [16]. Some recent development in medical diagnosis can also be encountered in [17-24]. Afterwards Zadeh [25] himself developed possibility theory which was thought to be

Electronic supplementary material The online version of this article (https://doi.org/10.1007/s42452-019-1871-8) contains supplementary material, which is available to authorized users.

Palash Dutta, palash.dtt@gmail.com | Department of Mathematics, Dibrugarh University, Dibrugarh 786004, India. 
better to treat uncertainty and further studied by acolyte researchers such as Dubois and Prade [26], Klir [27], Yager [28] etc. Furthermore, Dubois and Prade [29] studied mean value of fuzzy numbers, Ban [30] discussed fuzzy valued measure and conditional expectations of fuzzy numbers, Heilpern [31]studied expected value of fuzzy numbers, Carlsson and Fuller [32] developed possiblistic mean and variance of fuzzy numbers, Chen and Tan [33] further developed mean value and variance of multiplication of fuzzy numbers.

Nevertheless in the absence of self duality measure the earlier studies lead to the exaggeration of the reality. keeping this in mind, Liu and Liu [34] initiated a concept termed as credibility theory. Li and Liu [35] presented a sufficient and necessary condition for credibility measures. Further Liu and Liu [36] systematically studied and developed credibility theory. After that some extended studies on credibility theory can be observed in Liu [37], Zhou et al. [38], Yi et al. [39], Garai et al. [40].

Although various types of fuzzy variables are encountered, however, an exceptional and intricate fuzzy variable SEFV in terms of credibility theory is not deliberated yet. This paper presents an approach to derive possibility, necessity and credibility measure of the SEFV. Furthermore, expected value, variance, rational upper bound etc of SEFV are presented. Then ranking of SEFNs through expected value and variance is proposed. Finally, novelty and applicability has been exhibited by performing reliability analysis and medical diagnosis cases.

\section{Preliminaries}

Uncertainty is an important as well as unavoidable ingredient of decision making process. Depending on the nature and accessibility of data, information, uncertainty is generally modelled using fuzzy set, possibility theory and Credibility theory.

Definition Let $\Theta$ be a non-empty set, and $P$ the power set of $\Theta$, and Pos is possibility measure. Then, the triplet $(\Theta, P, P o s)$ is known as a possibility space. A fuzzy variable is a mapping from possibility space $(\Theta, P, P o s)$ to the set of real numbers $[37,39]$.

Definition Let $\zeta$ be a fuzzy variable defined on the credibility space $(\Theta, P, P o s)$. Then its membership function (MF) defined from the credibility measure is given by $[37,39]$

$\mu_{\zeta}(x)=\min (2 C r \zeta=1) ; x \in \mathbb{R}$.

Definition The $\alpha$-cut of a fuzzy variable $A$ is defined as
${ }^{\alpha} A=\left\{x \in X: \mu_{A}(x) \geq \alpha\right\}$.

Definition Let $A$ be a fuzzy variable, $\mu$ be the MF of $A$, and $r$ be any real number. Then, the possibility measure of $A$ is defined as [25]

$\operatorname{Pos}\{A \leq r\}=\sup _{x \leq r} \mu_{A}(x)$

Definition Let $A$ be a fuzzy variable, $\mu$ be the MF of $A$, and $r$ be any real number. Then, the possibility measure of $A$ is defined as [25]

$\operatorname{Nec}\{A \leq r\}=1-\sup _{x>r} \mu_{A}(x)$

Definition A credibility measure $(\mathrm{Cr})$ is a non-negative set function holds the following [35]

$$
\begin{aligned}
& \operatorname{Cr}(\Theta)=1 \\
& \operatorname{Cr}(A) \leq \operatorname{Cr}(B) \text { for whenever } A \subset B \\
& \operatorname{Cr}(A)+\operatorname{Cr}\left(A^{c}\right)=1 \text { for any } A \\
& \operatorname{Cr}\{\cup A\}=\operatorname{Sup}_{i} \operatorname{Cr}\left\{A_{i}\right\} \text { for any events } A_{i} \text { with } \\
& \underset{i}{\operatorname{Sup}} \operatorname{Cr}\left\{A_{i}\right\}<0.5
\end{aligned}
$$

If the fuzzy variable $A$ is given by its MF $\mu$, then

$\operatorname{Cr}(A \leq r)=\frac{1}{2}\left\{\operatorname{Sup}_{x \leq r} \mu_{A}(x)+1-\operatorname{Sup}_{x>r} \mu_{A}(x)\right\}, x, y \in \mathbb{R}$

Definition The credibility distribution $\Phi_{A}: \mathbb{R} \rightarrow[0,1]$ of a fuzzy variable $A$ is defined as [36]

$\Phi_{A}(x)=\operatorname{Cr}\{\theta \in \Theta: \zeta(\theta) \leq x\}$.

That is, the credibility that the fuzzy variable $\zeta$ takes a value less than or equal to $x$.

Definition The credibility density function of credibility distribution defined as $\phi_{A}: \mathbb{R} \rightarrow[0, \infty)$ of any fuzzy variable $A$, is a function such that [37]

$\phi_{A}(x)=\int_{\infty}^{x} \phi(y) d y, \forall x \in \mathbb{R}$

Definition [38] A credibility distribution $\Phi_{A}$ of a fuzzy variable $A$ is called regular if it is a continuous and strictly increasing function w.r.t $x$ such that $<\Phi_{A}<1$ and if $\lim _{x \rightarrow-\infty} \Phi_{A}=0$, and $\lim _{x \rightarrow \infty} \Phi_{A}=1$.

Definition [38] Let A be a fuzzy variable with a regular credibility distribution $\Phi_{A}$, then the inverse function $\Phi_{A}^{-1}$ is called the inverse credibility distribution of $A$. 


\section{Construction of semi-elliptic fuzzy variable}

Let's consider the general equation of the horizontal ellipse centred at $(a, b)$ is

$\frac{(x-a)^{2}}{h^{2}}+\frac{(y-b)^{2}}{k^{2}}=1$

To construct a normal semi-circular fuzzy variable (SEFV), it is needed to consider $b=0$ and $k=1$. i.e.,

$\frac{(x-a)^{2}}{h^{2}}+y^{2}=1$

Thus, the required membership function of the SEFV $A=S_{E}(a, h)$ is

$\mu_{A}(x)=\sqrt{1-\frac{(x-a)^{2}}{h^{2}}}, a-h \leq x \leq a+h$

where $a$ indicates the mean/core of the fuzzy variable while $h$ determines width of the fuzzy variable $A$.

The $\alpha$-cut of the SEFV $A=S_{E}(a, h)$ is

${ }^{\alpha} A=\left[a-h \sqrt{1-\alpha^{2}}, a+h \sqrt{1-\alpha^{2}}\right]$

Suppose $A=S_{E}(10,7)$ is SEFV representing a uncertain variable. The MF of the SEFV $A$ is

$\mu_{A}(x)=\sqrt{1-\frac{(x-10)^{2}}{49}}, 3 \leq x \leq 17$

The graphical representation of $A$ is depicted in Fig. 1.

\section{Possibility, necessity and credibility measures of SEFV}

In this section, the possibility measure, necessity measure and credibility measure of the SEFV are derived.

\subsection{Possibility measures of SEFV}

Suppose $A=S_{E}(a, h)$ is a SEFV.

Then, the possibility measure of $(A \leq x)$ and $(A \geq x)$ are respectively

$\operatorname{Pos}(A \leq x)= \begin{cases}1, & \text { if } x \geq a \\ \sqrt{1-\frac{(x-a)^{2}}{h^{2}}}, & \text { if } x<a\end{cases}$

$\operatorname{Pos}(A \geq x)= \begin{cases}1, & \text { if } x \leq a \\ \sqrt{1-\frac{(x-a)^{2}}{h^{2}},}, & \text { if } x>a\end{cases}$

The possibility measure of the SEFV $A=S_{E}(10,7)$ for $(A \leq x)$ and $(A \geq x)$ are depicted in Figs. 2 and 3 repectively.

\subsection{Necessity measures of SEFV}

Suppose $A=S_{E}(a, h)$ is a SEFV.

Then, the necessity measure of $A \leq x$ and $A \geq x$ are respectively

$\operatorname{Nec}(A \leq x)= \begin{cases}0, & \text { if } x \leq a, \\ \sqrt{1-\frac{(x-a)^{2}}{h^{2}},}, & \text { if } x>a,\end{cases}$

$\operatorname{Nec}(A \geq x)= \begin{cases}0, & \text { if } x \geq a, \\ \sqrt{1-\frac{(x-a)^{2}}{h^{2}},}, & \text { if } x<a,\end{cases}$

The necessity measure of the SEFV $A=S_{E}(10,7)$ for $(A \leq x)$ and $(A \geq x)$ are depicted in Figs. 4 and 5 repectively.

\subsection{Credibility measures of SEFV}

Suppose $A=S_{E}(a, h)$ is a SEFV.

Fig. 1 The SEFV $A=S_{E}(10,7)$

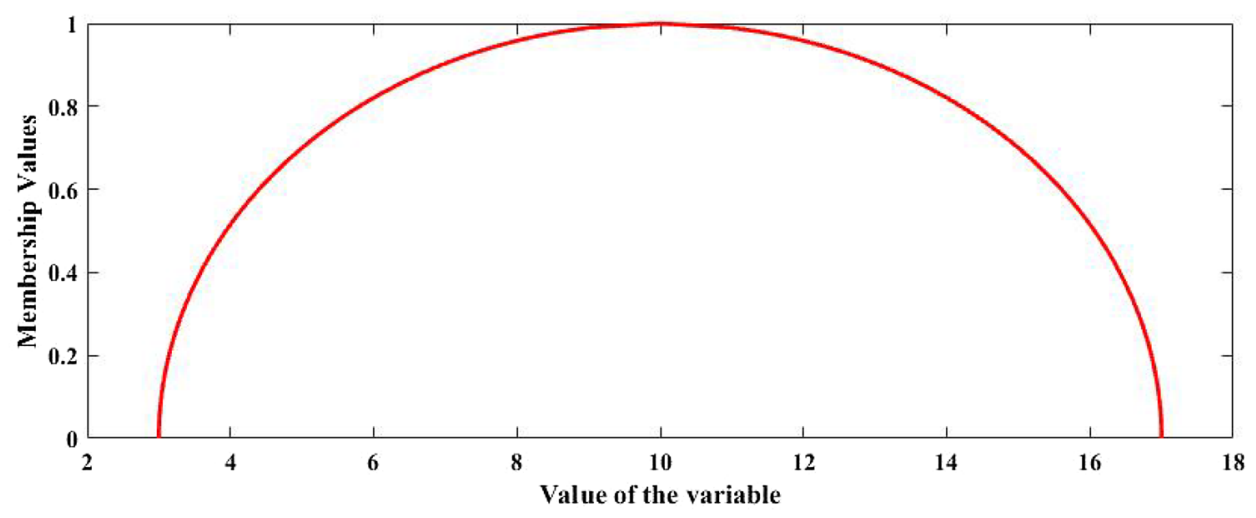


Fig. 2 The possibility measure of SEFV $A=S_{E}(10,7)$ for $(A \leq x)$

Fig. 3 The possibility measure of SEFV $A=S_{E}(10,7)$ for $(A \geq x)$

Fig. 4 The necessity measure of SEFV $A=S_{E}(10,7)$ for $(A \leq x)$

Fig. 5 The necessity measure of SEFV $A=S_{E}(10,7)$ for $(A \geq x$ )
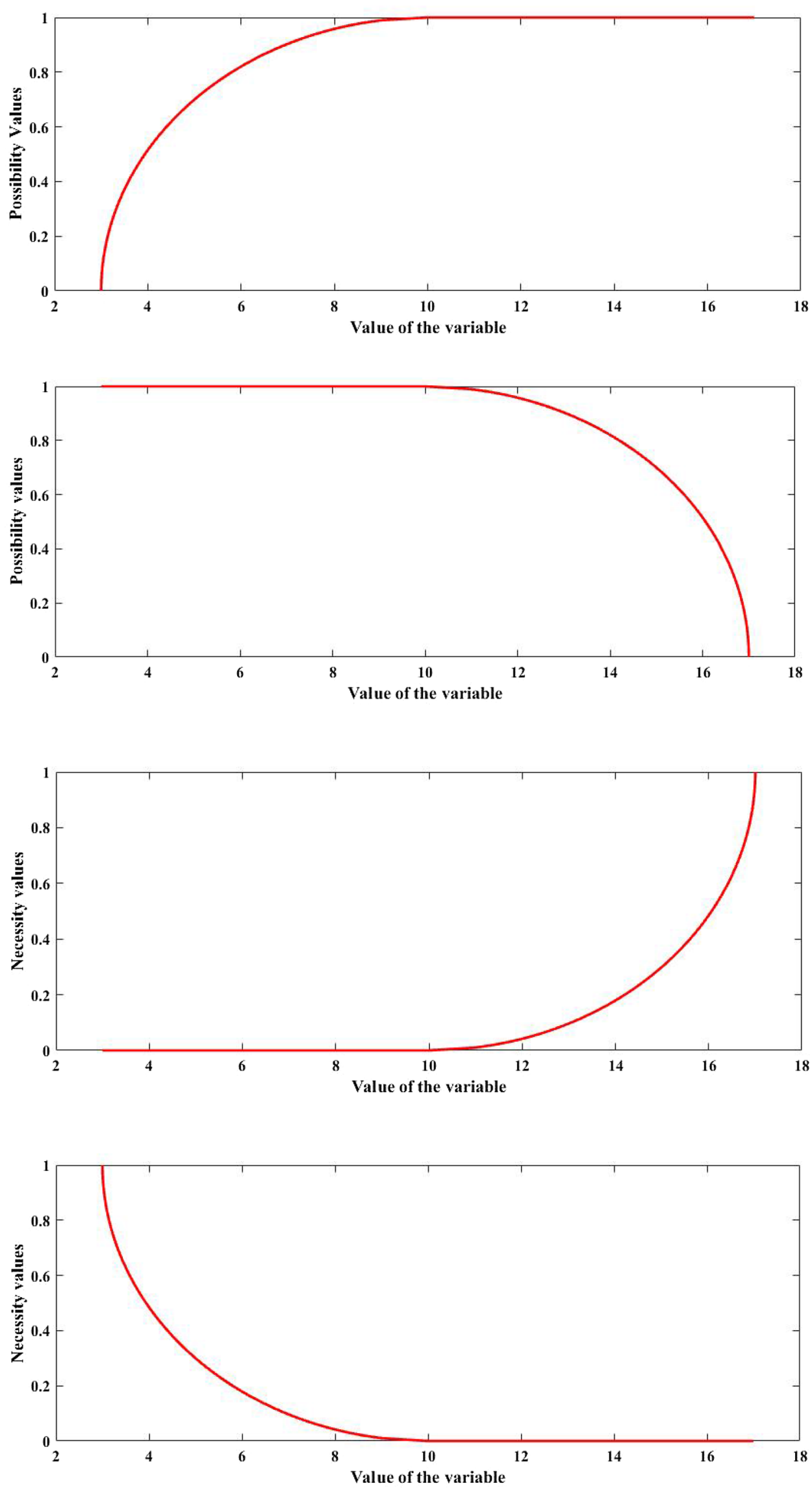
Then, the credibility measure of $A \leq x$ and $A \geq x$ are respectively

$\operatorname{Cr}(A \leq x)=\left\{\begin{array}{cl}\frac{1}{2} \sqrt{1-\frac{(x-a)^{2}}{h^{2}},} & \text { if } a-h \leq x \leq a, \\ 1-\frac{1}{2} \sqrt{1-\frac{(x-a)^{2}}{h^{2}}}, & \text { if } a \leq x \leq a+h,\end{array}\right.$

$\operatorname{Cr}(A \geq x)=\left\{\begin{array}{cl}1-\frac{1}{2} \sqrt{1-\frac{(x-a)^{2}}{h^{2}}}, & \text { if } a-h \leq x \leq a, \\ \frac{1}{2} \sqrt{1-\frac{(x-a)^{2}}{h^{2}}}, & \text { if } a \leq x \leq a+h,\end{array}\right.$

The necessity measure of the SEFV $A=S_{E}(10,7)$ for $(A \leq x)$ and $(A \geq x)$ are depicted in Figs. 6 and 7 repectively.

\subsection{Credibility distribution of SEFV}

As the credibility distribution $\Phi_{A}: \mathbb{R} \rightarrow[0,1]$ of a fuzzy variable $A$ is defined as $\Phi_{A}(x)=\operatorname{Cr}\{\theta \in \Theta: \zeta(\theta) \leq x\}$.

Hence, the credibility distribution of the SEFV $A=S_{E}(a, h)$ is
$\Phi_{A}(x)=\left\{\begin{array}{cl}\frac{1}{2} \sqrt{1-\frac{(x-a)^{2}}{h^{2}},}, & \text { if } a-h \leq x \leq a, \\ 1-\frac{1}{2} \sqrt{1-\frac{(x-a)^{2}}{h^{2}}}, & \text { if } a \leq x \leq a+h,\end{array}\right.$

The credibility distribution function of the SEFV $A=S_{E}(10,7)$ is

$\Phi_{A}(x)=\left\{\begin{array}{cl}\frac{1}{2} \sqrt{1-\frac{(x-10)^{2}}{49},}, & \text { if } 3 \leq x \leq 10, \\ 1-\frac{1}{2} \sqrt{1-\frac{(x-10)^{2}}{49}}, & \text { if } 10 \leq x \leq 17,\end{array}\right.$

\subsection{Inverse credibility distribution (ICD) of SEFV}

The credibility distribution of the SEFV $A=S_{E}(a, h)$ is

$\Phi^{-1}(\alpha)== \begin{cases}a-h \sqrt{1-4 \alpha^{2}}, & \text { if } 0 \leq \alpha \leq 0.5, \\ a+h \sqrt{1-4(1-\alpha)^{2}}, & \text { if } \quad 0.5 \leq \alpha \leq 1,\end{cases}$
Fig. 6 The credibility measure of SEFV $A=S_{E}(10,7)$ for $(A \leq x)$

Fig. 7 The credibility measure of SEFV $A=S_{E}(10,7)$ for $(A \geq x)$
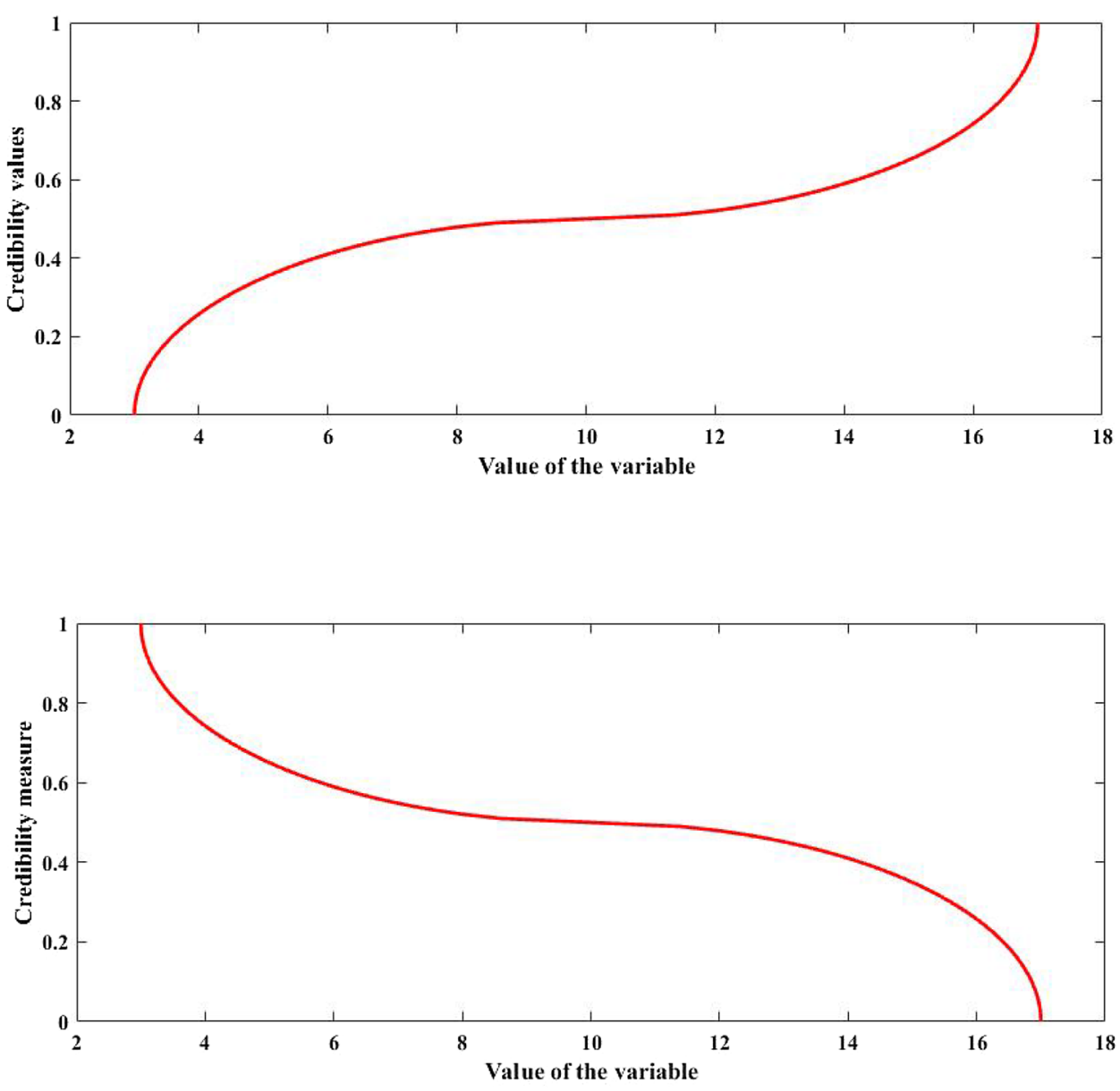

SN Applied Sciences 


\section{Expected value}

Using the idea of credibility distribution, Liu and Liu [34] provided the expected value of fuzzy variables. Zhou et al. [38] presented expected value of fuzzy variables via ICD.

\subsection{Expected value via credibility distribution}

If $A$ is a fuzzy variable then the expected value of $A$ in terms of credibility distribution is defined as [34]

$E(A)=\int_{0}^{\infty} \operatorname{Cr}\{A \geq r\} d r-\int_{\infty}^{0} \operatorname{Cr}\{A \leq r\} d r$

Then, the expected value of the SEFV $A=S_{E}(a, h)$ is

$$
\begin{aligned}
E(A)= & \int_{0}^{a-h} d r+\int_{a-h}^{a} \frac{1}{2} \sqrt{1-\frac{(r-a)^{2}}{h^{2}}} d r \\
& +\int_{a}^{a+h}\left\{1-\frac{1}{2} \sqrt{1-\frac{(r-a)^{2}}{h^{2}}}\right\} d r \\
= & (a-h)+h+\int_{a-h}^{a} \frac{1}{2} \sqrt{1-\frac{(r-a)^{2}}{h^{2}}} d r \\
& -\int_{a}^{a+h} \frac{1}{2} \sqrt{1-\frac{(r-a)^{2}}{h^{2}}} d r \\
= & a+\frac{\pi h}{8}-\frac{\pi h}{8} \\
= & a
\end{aligned}
$$

\subsection{Expected value via ICD}

If $A$ is a SEFV then the expected value of $A$ in terms of ICD is defined as [38]

$$
E[A]=\int_{0}^{1} \Phi^{-1}(\alpha) d \alpha
$$

Then, the expected value of the $\operatorname{SEFV} A=S_{E}(a, h)$ is

$$
\begin{aligned}
E(A)= & \int_{0}^{0.5}\left\{a-h \sqrt{1-4 \alpha^{2}}\right\} d \alpha \\
& +\int_{0.5}^{1}\left\{a+h \sqrt{1-4(1-\alpha)^{2}}\right\} d \alpha \\
= & a-\frac{h}{2} \int_{0}^{1} \sqrt{1-t^{2}} d t-\frac{h}{2} \int_{1}^{0} \sqrt{1-t^{2}} d t \\
= & a
\end{aligned}
$$

Thus, in both the approaches, it is obtained that the expected value of the SEFV $A$ is $e=a$.
Remark If $A=S_{E}\left(h_{1}, h_{2}\right)$ is a asymmetric SEFV where $h_{1}$ and $h_{2}$ are left spread and right spread of $A$. Then, the expected value of $A$ is

$E[A]=a+\frac{\pi}{8}\left(h_{2}-h_{1}\right)$.

\section{Variance}

In this section, variance of SEFV is calculated in terms of regular credibility distribution.

If $A$ is a fuzzy variable with expected value $e$, then the variance of $A$ is defined as [36]

$V[A]=E\left[(A-e)^{2}\right]$

It should be noted that if the expected value $e$ of the fuzzy variable is finite, then the variance satisfies

$V[A]=E\left[(A-e)^{2}\right]=\int_{0}^{+\infty} \operatorname{Cr}\left\{(A-e)^{2} \geq r\right\} d r$

\subsection{Variance of a SEFV}

To evaluate variance $V[A]$ of a SEFV $A$, it is needed to calculate the MF of $(A-e)^{2}$ first and to find MF of SEFV $A=S_{E}(a, h), \alpha$-cut technique is explored here.It is already obtained that the expected valued of SEFV is $e=a$

The $\alpha$-cut of the SEFV $A=S_{E}(a, h)$ is ${ }^{\alpha} A=\left[a-h \sqrt{1-\alpha^{2}}, a+h \sqrt{1-\alpha^{2}}\right]$.

The procedure is presented below.

$$
\begin{aligned}
\left({ }^{\alpha} A\right. & -a)^{2} \\
& =\left[\left(a-h \sqrt{1-\alpha^{2}}-a\right)^{2},\left(a+h \sqrt{1-\alpha^{2}}-a\right)^{2}\right] \\
& =\left[\left(-h \sqrt{1-\alpha^{2}}\right)^{2},\left(h \sqrt{1-\alpha^{2}}\right)^{2}\right] \\
& =\left[h^{2}\left(1-\alpha^{2}\right),\left(h^{2}\left(1-\alpha^{2}\right]\right.\right.
\end{aligned}
$$

Now, taking $x=h^{2}\left(1-\alpha^{2}\right)$ gives $\alpha=\sqrt{1-\frac{x}{h^{2}}}, 0 \leq x \leq h^{2}$

Thus, the MF of $(A-e)^{2}$ is

$\mu_{(A-e)^{2}}(x)=\sqrt{1-\frac{x}{h^{2}}}, 0 \leq x \leq h^{2}$.

Since $C r\left\{(A-e)^{2}<r\right\}=\frac{1}{2}\left\{\operatorname{Sup}_{x<r} \mu_{(A-e)^{2}}(x)+1-\sup _{x \geq r} \mu_{(A-e)^{2}}(x)\right\}$

$\therefore C r\left\{(A-e)^{2}<r\right\}=\frac{1}{2}\left\{1+1-\sqrt{1-\frac{x}{h^{2}}}\right\}, 0 \leq x \leq h^{2}$. 
Again, $\operatorname{Cr}\left\{(A-e)^{2} \geq r\right\}=1-\operatorname{Cr}\left\{(A-e)^{2}<r\right\}$. Hence,

$C r\left\{(A-e)^{2} \geq r\right\}=\left\{\begin{array}{l}1-C r\left\{(A-e)^{2}<r\right\}, \quad 0 \leq x \leq h^{2} . \\ 0, \quad r>h^{2}\end{array}\right.$

Then, the variance of SEFV $A=S_{E}(a, h)$ is

$V[A]$

$$
\begin{aligned}
& =\int_{0}^{\infty} \operatorname{Cr}\left\{(A-e)^{2} \geq r\right\} d r \\
& =\int_{0}^{h^{2}} \frac{1}{2} \sqrt{1-\frac{r}{h^{2}}} d r \\
& =\frac{h^{2}}{3}
\end{aligned}
$$

For example, the variance of the SEFV $A=S_{E}(10,7)$ is $\frac{7^{2}}{3}=16.33$.

Remark If the width $h$ of a SEFV is unit then SEFV will represent a semi-circular fuzzy variable (SCFV). Then, it can be derived that for all SCFV the expected value is 0.33 .

\section{Rational upper bound of the variance}

Yi et al. [39] derived the concept of rational upper bounded of the variance (RUBV) along with some definitions and results in terms of credibility distribution and ICD.

Definition Let $A$ be a fuzzy variable with credibility distribution $\Phi$ and finite expected value $e$. The RUBV is defined as [39]

$\bar{V}[A]=\int_{0}^{\infty}(1-\Phi(e+\sqrt{x}) \Phi(e-\sqrt{x}))$

Definition Let $A$ be a fuzzy variable with credibility distribution $\Phi$. If the expected value is $e$, then RUBV is evaluated as [39]

$\bar{V}[A]=\int_{0}^{1}\left(\Phi^{-1}(\alpha)-e\right)^{2} d \alpha$

Corollary The RUBV of SEFV depends on the width $h$ and it is $\frac{2 h^{2}}{3}$.

Consider the SEFV A $=S_{E}(a, h)$.

$$
\begin{aligned}
\int_{0}^{0.5} & h^{2}\left(1-4 \alpha^{2}\right) d \alpha+\int_{0.5}^{1} h^{2}\left(1-4(1-\alpha)^{2}\right) d \alpha \\
= & h^{2}+h^{2} \int_{0}^{0.5} 4 \alpha^{2} d \alpha-h^{2} i n t_{0.5}^{1} 4(1-\alpha)^{2} d \alpha \\
= & h^{2}-\frac{h^{2}}{2} \int_{0}^{1} t^{2} d t+\frac{h^{2}}{2} \int_{1}^{0} t^{2} d t \\
= & h^{2}-h^{2} \int_{0}^{1} t^{2} d t \\
= & h^{2}-\frac{h^{2}}{3} \\
= & \frac{2 h^{2}}{3}
\end{aligned}
$$

Corollary Let $A=S_{E}(a, h)$ be SEFV. Then, $\bar{V}[A]=2 V[A]$.

Remark The SCFV also satisfies the above corollary and it is also observed that for all SCFV, the RUBV is 0.66 .

Corollary Suppose $A=S_{E}\left(a_{1}, h_{1}\right)$ and $B=S_{E}\left(a_{2}, h_{2}\right)$ are two SRFV.

Then, $\bar{V}[A+B] \leq 2(\bar{V}[A]+\bar{V}[B]$.

For the two SEFV $A$ and $B$, the ICV of $A+B$ is

$$
\begin{aligned}
& \Phi^{-1}(\alpha) \\
& \quad=\Phi_{A}^{-1}(\alpha)+\Phi_{B}^{-1}(\alpha) \\
& \quad=\left\{\begin{array}{l}
\left(a_{1}+b_{1}\right)-\left(h_{1}+h_{2}\right) \sqrt{1-4 \alpha^{2}}, \alpha \leq 0.5 \\
\left(a_{1}+b_{1}\right)+\left(h_{1}+h_{2}\right) \sqrt{1-4(1-\alpha)^{2}}, \alpha>0.5
\end{array}\right.
\end{aligned}
$$

Now,

$$
\begin{aligned}
\bar{V}[A & +B] \\
= & \int_{0}^{0.5}\left[\left(a_{1}+b_{1}\right)-\left(h_{1}+h_{2}\right) \sqrt{1-4 \alpha^{2}} d \alpha\right. \\
& +\int_{0.5}^{1}\left(a_{1}+b_{1}\right)+\left(h_{1}+h_{2}\right) \sqrt{1-4(1-\alpha)^{2}} d \alpha \\
= & \frac{2\left(h_{1}+h_{2}\right)^{2}}{3} .
\end{aligned}
$$

Again, $\bar{V}[A]=\frac{2\left(h_{1}\right)^{2}}{3}$ and $\bar{V}[B]=\frac{2\left(h_{2}\right)^{2}}{3}$.

Since, $\frac{2\left(h_{1}+h_{2}^{3}\right)^{2}}{3} \leq \frac{2\left(h_{1}\right)^{2}}{3}+\frac{2\left(h_{2}^{3}\right)^{2}}{3}$.

Consequently, $\bar{V}[A+B] \leq 2(\bar{V}[A]+\bar{V}[B]$.

Corollary Suppose $A=S_{E}\left(a_{1}, h_{1}\right)$ and $B=S_{E}\left(a_{2}, h_{2}\right)$ are two SRFVs.

$$
\begin{aligned}
& \text { SRFVs. } \sqrt{\bar{V}[A+B]}=\sqrt{(\bar{V}[A]}+\sqrt{\bar{V}[B]} . \\
& \text { Since } \sqrt{\bar{V}[A+B]}=\sqrt{\frac{2\left(h_{1}+h_{2}\right)^{2}}{3}} \text { and } \sqrt{\bar{V}[A]}=\sqrt{\frac{2 h_{1}^{2}}{3}}, \\
& \sqrt{\bar{V}[B]}=\sqrt{\frac{2 h_{2}^{2}}{3}} .
\end{aligned}
$$




$$
\text { Thus, } \sqrt{\bar{V}[A+B]}=\sqrt{\overline{(V}[A]}+\sqrt{\bar{V}[B]} \text {. }
$$

\section{Arithmetic on SEFVs}

In this section, basic operations on SEFVs are reviewed and adopted from [41].

Suppose $A=S_{E}(a, h)$ and $B=S_{E}(b, k)$ are two SEFVs defined on a universe of discourse $X$.

\subsection{Addition}

The membership function of $A+B$ is

$$
\begin{aligned}
\mu_{(A+B)}= & \sqrt{1-\left\{\frac{x-(a+b)}{h+k}\right\}^{2}}, \\
& x \in[(a-h)+(b-k),(a+h)+(b+k)]
\end{aligned}
$$

\subsection{Subtraction}

The membership function of $A-B$ is

$$
\begin{aligned}
\mu_{(A-B)}= & \sqrt{1-\left\{\frac{x-(a-b)}{h+k}\right\}^{2}}, \\
& x \in[(a-h)-(b+k),(a+h)-(b-k)]
\end{aligned}
$$

\subsection{Multiplication}

The membership function of $A B$ is

$$
\begin{gathered}
\mu_{A B}(x)=\sqrt{1-\left\{\frac{(a k+b h)-\sqrt{(a k+b h)^{2}-4 h k(a b-x)}}{2 h k}\right\}}, \\
x \in(a-h)(b-k) \leq x \leq(a+h)(b+k)
\end{gathered}
$$

\subsection{Division}

The membership function of $A$ / $B$ is

$$
\mu_{\bar{B}}(x)=\sqrt{1-\left\{\frac{a-x b}{h+x k}\right\}}, \frac{a-h}{b+k} \leq x \leq \frac{a+h}{b-k}
$$

\section{Rank of SEFVs}

In this section, ranking of two SEFVs are defined based on the expected value and variance of the SEFVs. Suppose $A=S_{E}\left(a_{1}, h_{1}\right)$ and $B=S_{E}\left(a_{2}, h_{2}\right)$ are two SEFVs.

Then rank of $A$ and $B$ are defined as
I. $A \leq B$ if $E[A] \leq E[B]$.

II. If $E[A]=E[B]$ then

$$
A \leq B \text { if } V[A] \geq V[B]
$$

Example 9.1 Suppose $A=[-4,0,4]$ and $B=[-2,0,2]$ are two fuzzy variables adopted from [42]. It is observed that the approaches [42-47] fail to compare the fuzzy variables. Reforming these fuzzy variables to SEFVs as $A=S_{E}(0,4)$ and $B=S_{E}(0,2)$ and applying the present approach it is obtained that $A \leq B$ which is consistent with human intuitions. Here, $E[A]=E[B]=0$, but $V[A]=5.33 \geq V[B]=1.33$ and consequently, it can be adopted that $A \leq B$. A details comparison has been presented in Table 1.

Example 9.2 Consider the fuzzy variables $A=[0.2,0.5,0.8]$ and $B=[0.4,0.5,0.6]$. The approaches $[44,47-49,51]$ are not applicable to distinguish $A$ and $B$ while [52-54] and [50] produces illogical output. Here also the general or human intuition is that $A \leq B$. Applying the present approach we obtain $E[A]=E[B]=0.5$, but $V[A]=0.1 \geq V[B]=0.033$ which gives $A \leq B$. A detail discussion is presented in Table 2.

From the above analysis it can be opined that the present ranking approach has the capability to overcome the drawbacks of the existing approaches.

\section{Application of SEFVs}

In this segment, application of SEFVs are performed in structural reliability analysis and medical diagnosis. For structural reliability arithmtic of SEFVs are taken into consideration while in medical diagnosis arithmetic as well as ranking of SEFVs are adopted.

Table 1 Ranking of fuzzy variables for example 8.1

\begin{tabular}{llll}
\hline Approaches & $A$ & $B$ & Result \\
\hline Abbasbandy et al. [42] & 0 & 0 & $A \sim B$ \\
Wang [43] & 0 & 0 & $A \sim B$ \\
Asady [44] & 0.9 & 0.9 & $A \sim B$ \\
Asady [45] & 0 & 0 & $A \sim B$ \\
Asady [46] & 0.444 & 0.444 & $A \sim B$ \\
Abbasbandy and Hajjari [47] & 0.117 & 0.074 & $A>B$ \\
Present approach & $E[A]=0.5$ & $E[B]=0.5$ & \\
& $V[A]=5.33$ & $V[B]=1.33$ & $A \leq B$ \\
\hline
\end{tabular}


Table 2 Ranking of fuzzy variables for example 8.2

\begin{tabular}{lllll}
\hline Approaches & Decision-level & $A$ & $B$ & Result \\
\hline Yager [48] & & 0.5 & 0.5 & $A \sim B$ \\
Abbhasbandy and Hajjari [47] & & 0.5 & 0.5 & $A \sim B$ \\
Asady [44] & 0.374 & 0.374 & $A \sim B$ \\
Chen and Sanguansat [51] & 0.5 & 0.5 & $A \sim B$ \\
Chen et al. [49] & 0.444 & 0.444 & $A \sim B$ \\
Vincent et al. [52, 53] & 0.117 & 0.074 & $A>B$ \\
Vincent and Dat [54] & 0.45 & 0.35 & $A>B$ \\
Rezvani [50] & $\alpha=1$ & 0.078 & 0.062 & $A>B$ \\
Present Approach & & $E[A]=0.5$ & $E[B]=0.5$ & \\
& & $V[A]=0.1$ & $V[B]=0.033$ & $A \leq B$ \\
\hline
\end{tabular}

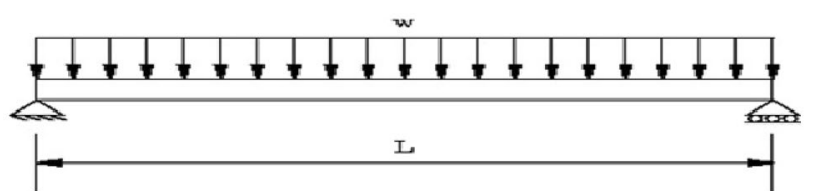

Fig. 8 Beam associated with its bending moment

\subsection{Application in structural reliability}

Depictions of the elements of structural analysis are more often SEFVs in some circumstances. In such circumstances structural failure can be evaluated using arithmetic of SEFVs. Consider the following problem of structural failure adopted from Dutta [3].

Example 10.1 Suppose a beam of height $h=9 \mathrm{~mm}$, length $L=1250 \mathrm{~mm}$ and the force density $f=78.5 \times 10^{-5} \mathrm{kN} / \mathrm{mm}^{3}$. The load $w$, breadth of the beam $b$ and ultimate bending moment $M_{O}$ are uncertain input variables represented by SEFVs where $w=S_{E}(400,15) k N, b=S_{E}(40,5)$ and $M_{o}=S_{E}\left(2.05 \times 10^{5}, 0.05 \times 10^{5}\right) \mathrm{kN}-\mathrm{mm}$. which is depicted in Fig. 8.

The limit state function is $g\left(b, f, h, w, M_{o}, L\right)=M_{o}-\left(\frac{w L}{4}+\frac{f b h L^{2}}{8}\right)$.

It is needed to evaluate the structural failure of the beam.

Applying the arithmetic of SEFVs on the problem the value of structural reliability or $g$ is obtained as $S_{E}\left(0.248 \times 10^{5}, 0.1658 \times 10^{5}\right)$.

\subsection{Application in medical diagnosis}

It is observed that patient's explanations, medical information even medical assessment process tainted with imprecision/vagueness/uncertainty. On the other hand, knowledge base correlating the symptom-disease relationship
Table 3 Patient-symptom relation

\begin{tabular}{llll}
\hline$R_{1}$ & $S_{1}$ & $S_{2}$ & $S_{3}$ \\
\hline$P_{1}$ & $S_{E}(3,1)$ & $S_{E}(6,1)$ & $S_{E}(2,1)$ \\
$P_{2}$ & $S_{E}(7,1)$ & $S_{E}(2,1)$ & $S_{E}(4,2)$ \\
$P_{3}$ & $S_{E}(2,1)$ & $S_{E}(3,1)$ & $S_{E}(7,2)$ \\
\hline
\end{tabular}

Table 4 Symptom-disease relation

\begin{tabular}{llll}
\hline$R_{2}$ & $D_{1}$ & $D_{2}$ & $D_{3}$ \\
\hline$S_{1}$ & $S_{E}(6,2)$ & $S_{E}(4,2)$ & $S_{E}(3,1)$ \\
$S_{2}$ & $S_{E}(2,1)$ & $S_{E}(6,1)$ & $S_{E}(2,1)$ \\
$S_{3}$ & $S_{E}(3,1)$ & $S_{E}(2,1)$ & $S_{E}(7,2)$ \\
\hline
\end{tabular}

encompasses of ambiguity and uncertainty in medical assessment process. Accordingly to deal with such uncertainties FST is being adopted and became most demanding area in medical assessment process. Here, SEFVs are considered to represent uncertain information.

Consider the Patient-symptom and Symptom-disease relations presented in Tables 3 and 4, respectively.

Using the multiplication and addition of SEFVs the resultant Patient-disease relation is evaluated and presented in Table 5. Then, ranking of SEFVs is adopted to obtain crisp values of resultant SEFVs and presented in Table 6. It should be noted that maximum value in each row indicates that the patient is likely to have the disease. Here, $\left\{P_{1}, P_{2}, P_{3}\right\},\left\{S_{1}, S_{2}, S_{3}\right\}$ and $\left\{D_{1}, D_{2}, D_{3}\right\}$ are the set of patients, symptoms and diseases, respectively. From Table 6, it is clear that the maximum value (the bold value) in 1 st row is 59.0686 which associates patient $P_{1}$ and

Table 5 Patient-Disease relation

\begin{tabular}{llll}
\hline$R$ & $D_{1}$ & $D_{2}$ & $D_{3}$ \\
\hline$P_{1}$ & $S_{E}(36,17,40)$ & $S_{E}(52,20,38)$ & $S_{E}(35,19,33)$ \\
$P_{2}$ & $S_{E}(58,29,35)$ & $S_{E}(48,29,28)$ & $S_{E}(53,30,28)$ \\
$P_{3}$ & $S_{E}(39,23,29)$ & $S_{E}(40,23,27)$ & $S_{E}(61,32,27)$ \\
\hline
\end{tabular}


Table 6 Crisp values of the patient-disease relation

\begin{tabular}{llll}
\hline$R$ & $D_{1}$ & $D_{2}$ & $D_{3}$ \\
\hline$P_{1}$ & 45.0321 & $\mathbf{5 9 . 0 6 8 6}$ & 40.4978 \\
$P_{2}$ & $\mathbf{6 0 . 3 5 6 2}$ & 47.6073 & 52.2146 \\
$P_{3}$ & 41.3562 & 41.5708 & $\mathbf{5 9 . 0 3 6 5}$ \\
\hline
\end{tabular}

disease $D_{2}$. That is, patient $P_{1}$ is likely to have the disease $D_{2}$. Similarly, from 2nd and 3rd row (bold values in the Table 6) it can be opined that patient $P_{2}$ is suffering from disease $D_{1}$ and patient $P_{3}$ is suffering from disease $D_{3}$.

\section{Conclusions}

Uncertainty is an integral part of real world problems such as reliability assessment as well as medical diagnosis problems. To cope with uncertainty a handful number of fuzzy variables are demonstrated yet in literature. However, a special complicated fuzzy variable SEFV is not deliberated well and in this regard here SEFV has been introduced in terms of credibility theory first. Then, some important properties such as possibility measure, necessity measure, credibility measure, credibility distribution and ICD were presented. Afterwards, investigations on expected value of SEFV using credibility distribution and ICD along with variance and RUVB of SEFV have been performed and established relationship between them. Another important concept ranking of SEFVs is introduced based on expected value of SEFV and if it fails then variance of SEFVs concept has been utilized to evaluate order of SEFVs. Comparative numerical illustrations have been presented where results of existing methods and present method were compared and exhibited that present method smoothly over come the limitations of earlier methods. Finally, reliability analysis has been performed using arithmetic of SEFV while a medical diagnosis is performed using arithmetic and rank of SEFVs as well. The present model successfully solves both the problems which exhibits the novelty and applicability of the present model. However, the limitation of this present model is that it can't work properly when asymmetric SEFVs come into picture. Therefore, as an extension of this work, asymmetric SEFV will be investigated.

\section{Compliance with ethical standards}

Conflict of interest The authors declare that they have no conflict of interest.

Ethical approval This article does not contain any studies with human or animal subjects.

\section{References}

1. Zadeh LA (1965) Fuzzy sets, Inform. Control 8:338-356

2. Mohanta DK (2010) Fuzzy reliability evaluations in electric power systems. In: Panigrahi BK, Abraham A, Das S (eds) Computational intelligence in power engineering. Springer, Berlin, pp 103-130

3. Dutta P (2019) Structural reliability analysis with inverse credibility distributions. New Math Nat Comput 15(01):47-63

4. Rahimi T, Jahan H, Blaabjerg F, Bahman A, Hosseini S (2019) Fuzzy-logic-based mean time to failure (MTTF) analysis of interleaved DC-DC converters equipped with redundant-switch configuration. Appl Sci 9(1):88

5. Abdolshah M, Samavi A, Khatibi SA, Mamoolraftar M (2019) A review of systems reliability analysis using fuzzy logic. In: Ram $M$ (ed) Advanced fuzzy logic approaches in engineering science. IGI Global, pp 362-377

6. Li H, Nie Z (2018) Structural reliability analysis with fuzzy random variables using error principle. Eng Appl Artif Intell 67:9199. https://doi.org/10.1016/j.engappai.2017.08.015

7. Gao P, Xie L, Hu W, Liu C, Feng J (2018) Dynamic fuzzy reliability analysis of multistate systems based on universal generating function. Math Probl Eng. https://doi.org/10.1109/ACCES S.2019.2941508

8. Ebrahimnejad A, Jamkhaneh EB (2018) System reliability using generalized intuitionistic fuzzy Rayleigh lifetime distribution. Appl Appl Math 13(1):97-113

9. Kumar A, Ram M (2018) System reliability analysis based on weibull distribution and hesitant fuzzy set. Int J Math Eng Manag Sci 3(4):513-521

10. Olawoyin R (2017) Risk and reliability evaluation of gas connector systems using fuzzy theory and expert elicitation. Cogent Eng 4(1):1372731

11. Jamali S, Bani MJ (2017) Application of fuzzy assessing for reliability decision making. In: Proceedings of the world congress on engineering and computer science (2017)

12. Lacas E, Santolay JL, Biedermann A (2016) Obtaining sustainable production from the product design analysis. J Clean Prod 139:706-716. https://doi.org/10.1016/j.jclepro.2016.08.078

13. Zhi-gang L, Jun-gang Z, Bo-ying L (2016) Research on reliability evaluation method of complex multistate system based on fuzzy fault tree. Int Conf Fuzzy Theory Appl (Fuzzy). https://doi. org/10.1109/iFUZZY.2016.8004957

14. Rachid B, Hafaifa A, Hadroug N, Boumehraz M (2016) Reliability evaluation based on a fuzzy expert system: centrifugal pump application. Stud Inf Control. https://doi.org/10.24846/v25i2 y201605

15. Rizv S, Singh V, Khan A (2016) Fuzzy logic based software reliability quantification framework: early stage perspective (FLSRQF). Procedia Comput Sci 89:359-368. https://doi.org/10.1016/j. procs.2016.06.083

16. Dutta $P$ (2017) Decision making in medical diagnosis via distance measures on interval valued fuzzy sets. Int J Syst Dyn Appl 6(4):63-83

17. Dutta $P$ (2018) Medical diagnosis based on distance measures between picture fuzzy sets. Int J Fuzzy Syst Appl 7(4):15-36

18. Dutta P (2018) Medical diagnosis via distances measures between credibility distributions. Int J Decis Support Syst Technol 10(4):1-16

19. Talukdar P, Dutta $P$ (2018) Disease diagnosis using an advanced distance measure for Intuitionistic Fuzzy Sets. Int Res J Microbiol 7(2):029-042

20. Dutta P, Dash SR (2018) Medical decision making via the arithmetic of generalized triangular fuzzy numbers. Open Cybern Syst. https://doi.org/10.2174/1874110X01812010001 
21. Dutta P, Limboo B (2017) Bell-shaped fuzzy soft sets and their application in medical diagnosis. Fuzzy Inf Eng 9(1):67-91

22. Dutta $P$ (2017) Medical diagnosis via distance measures on picture fuzzy sets. AMSE J AMSE IIETA Publ 2017 Ser Adv A 54(2):657-672

23. Dutta P, Doley D (2020) Medical diagnosis under uncertain environment through bipolar-valued fuzzy sets. In: Gupta M, Konar D, Bhattacharyya S, Biswas S (eds) Computer vision and machine intelligence in medical image analysis. Springer, Singapore, pp 127-135

24. Dutta $P$ (2020) A straightforward advanced ranking approach of fuzzy numbers. In: Satapathy SC,Bhateja V, Mohanty JR, Udgata SK (eds) Smart intelligent computing and applications. Springer, Singapore, pp 475-483

25. Zadeh LA (1978) Fuzzy set as a basis for a theory of possibility. Fuzzy Set Syst 1:3-28

26. Dubois D, Prade H (1988) Possibility theory. Plenum Press, New York

27. Klir JG (1992) On fuzzy set interpretation of possibility theory. Fuzzy Sets Syst 108:263-273

28. Yager RR (1992) On the specificity of a possibility distribution. Fuzzy Sets Syst 50:279-292

29. Dubois D, Prade H (1987) The mean value of a fuzzy number. Fuzzy Sets Syst 24:279-300

30. Ban J (1990) Radon-Nikodým theorem and conditional expectation of fuzzy-valued measures and variables. Fuzzy Sets Syst 34(3):383-392

31. Heilpern $S$ (1992) The expected value of a fuzzy number. Fuzzy Sets Syst 47:81-86

32. Carlsson C, Fuller R (2001) On possibilistic mean and variance of fuzzy number. Fuzzy Sets Syst 122:315-326

33. Chen W, Tan S (2009) On the possibilistic mean value and variance of multiplication of fuzzy numbers. J Comput Appl Math 232(2):327-334

34. Liu B, Liu YK (2002) Expected value of fuzzy variable and fuzzy expected value model. IEEE Trans Fuzzy Syst 10(4):445-45

35. Li X, Liu B (2006) A sufficient and necessary condition for credibility measures. Int J Uncertain Fuzziness Knowl Based Syst 14(5):527-535

36. Liu B (2006) A survey of credibility theory. Fuzzy Optim Decis Mak 5(4):387-408

37. Liu B (2004) Uncertainty theory: a branch of mathematics for modeling human uncertainty. Springer, Berlin

38. Zhou J, Yang F, Wang K (2015) Fuzzy arithmetic on LR fuzzy numbers with applications to fuzzy programming. J Intell Fuzzy Syst. https://doi.org/10.3233/IFS-151712

39. Yi X, Miao Y, Zhou J, Wang Y (2016) Some novel inequalities for fuzzy variables on the variance and its rational upper bound. $J$ Inequal Appl 1:41
40. Garai T, Chakraborty D, Roy TK (2017) Expected value of exponential fuzzy number and its application to multi-item deterministic inventory model for deteriorating items. J Uncertain Anal Appl 5(1):8

41. Dutta P, Saikia B (2019) Arithmetic operations on normal semi elliptic intuitionistic fuzzy numbers and their application in decision-making. Granul Comput. https://doi.org/10.1007/ s41066-019-00175-5

42. Abbasbandy S, Nuraei R, Ghanbari M (2013) Revision of sign distance method for ranking of fuzzy numbers. Iran J Fuzzy Syst 10(4):101-117

43. Wang ZX, Liu YJ, Fan ZP, Feng $B$ (2009) Ranking L-R fuzzy number based on deviation degree. Inf Sci 179(13):2070-2077

44. Asady B (2010) The revised method of ranking LR fuzzy number based on deviation degree. Expert Syst Appl 37(7):5056-5060

45. Asady B, Zendehnam A (2007) Ranking fuzzy numbers by distance minimization. Appl Math Model 31(11):2589-2598

46. Asady B (2011) Revision of distance minimization method for ranking of fuzzy numbers. Appl Math Model 35(3):1306-1313

47. Abbasbandy S, Hajjari T (2009) A new approach for ranking of trapezoidal fuzzy numbers. Comput Math Appl 57(3):413-419

48. Yager RR (1979) Ranking fuzzy subsets over the unit interval. In: 1978 IEEE conference on decision and control including the 17th symposium on adaptive processes, IEEE, pp 1435-1437

49. Chen SM, Munif A, Chen GS, Liu HC, Kuo BC (2012) Fuzzy risk analysis based on ranking generalized fuzzy numbers with different left heights and right heights. Expert Syst Appl 39(7):6320-6334

50. Rezvani S (2015) Ranking generalized exponential trapezoidal fuzzy numbers based on variance. Appl Math Comput 262:191-198

51. Chen SM, Sanguansat K (2011) Analyzing fuzzy risk based on a new fuzzy ranking method between generalized fuzzy numbers. Expert Syst Appl 38(3):2163-2171

52. Vincent FY, Chi HTX, Shen CW (2013) Ranking fuzzy numbers based on epsilon-deviation degree. Appl Soft Comput 13(8):3621-3627

53. Vincent FY, Chi HTX, Dat LQ, Phuc PNK, Shen CW (2013) Ranking generalized fuzzy numbers in fuzzy decision making based on the left and right transfer coefficients and areas. Appl Math Model 37(16-17):8106-8117

54. Vincent FY, Dat LQ (2014) An improved ranking method for fuzzy numbers with integral values. Appl Soft Comput 14:603-608

Publisher's Note Springer Nature remains neutral with regard to jurisdictional claims in published maps and institutional affiliations. 